ACUTE CORONARY SYNDROMES

\title{
Intervention in acute coronary syndromes: do patients undergo intervention on the basis of their risk characteristics? The Global Registry of Acute Coronary Events (GRACE)
}

\author{
K A A Fox, F A Anderson Jr, O H Dabbous, P G Steg, J López-Sendón, F Van de Werf, A Budaj, \\ E P Gurfinkel, S G Goodman, D Brieger, on behalf of the GRACE investigators
}

Heart 2007;93:177-182. doi: 10.1136/hrt.2005.084830

See end of article for authors' affiliations

Correspondence to: Professor Keith A A Fox, Cardiovascular Research, University of Edinburgh, Chancellor's Building, 49 Little France Crescent, Edinburgh EH16 4SB, UK; k.a.a.fox@ed.ac.uk

Accepted 24 May 2006

Published Online First

6 June 2006

\begin{abstract}
Objective: To determine whether revascularisation is more likely to be performed in higher-risk patients and whether the findings are influenced by hospitals adopting more or less aggressive revascularisation strategies.

Methods: GRACE (Global Registry of Acute Coronary Events) is a multinational, observational cohort study. This study involved 24189 patients enrolled at 73 hospitals with on-site angiographic facilities.

Results: Overall, $32.5 \%$ of patients with a non-ST elevation acute coronary syndrome (ACS) underwent percutaneous coronary intervention ( $\mathrm{PCl} ; 53.7 \%$ in ST segment elevation myocardial infarction (STEMI)) and 7.2\% underwent coronary artery bypass grafting (CABG; $4.0 \%$ in STEMI). The cumulative rate of in-hospital death rose correspondingly with the GRACE risk score (variables: age, Killip class, systolic blood pressure, ST segment deviation, cardiac arrest at admission, serum creatinine, raised cardiac markers, heart rate), from $1.2 \%$ in low-risk to $3.3 \%$ in medium-risk and $13.0 \%$ in high-risk patients (c statistic $=0.83$ ). $\mathrm{PCl}$ procedures were more likely to be performed in low- (40\% non-STEMI, 60\% STEMI) than medium- $(35 \%, 54 \%)$ or highrisk patients $(25 \%, 41 \%)$. No such gradient was apparent for patients undergoing CABG. These findings were seen in STEMI and non-ST elevation ACS, in all geographical regions and irrespective of whether hospitals adopted low (4.2-33.7\%, $\mathrm{n}=7210$ observations), medium (35.7-51.4\%, $\mathrm{n}=7913$ observations) or high rates $(52.6-77.0 \%, \mathrm{n}=8942$ observations) of intervention.

Conclusions: A risk-averse strategy to angiography appears to be widely adopted. Proceeding to $\mathrm{PCl}$ relates to referral practice and angiographic findings rather than the patient's risk status. Systematic and accurate risk stratification may allow higher-risk patients to be selected for revascularisation procedures, in contrast to current international practice.
\end{abstract}

\section{METHODS}

Full details of the GRACE methods have been published. ${ }^{11-13}$ GRACE is designed to reflect an unbiased population of patients with ACS, irrespective of geographical region. A total of 106 hospitals from 14 countries in North and South America,
Europe, Australia and New Zealand have participated in this prospective observational study.

Patients entered in the study had to be at least 18 years old and alive at the time of hospital presentation, be admitted with ACS as the presumptive diagnosis (with symptoms consistent with acute ischaemia) and have at least one of the following: ECG changes consistent with ACS, serial increases in serum biochemical markers of cardiac necrosis and documentation of coronary artery disease. The qualifying ACS must not have been precipitated by significant non-cardiovascular co-morbidity (for example, trauma or surgery). At about six months after hospital discharge, patients were followed up to ascertain the occurrence of selected long-term study outcomes. Where required, study investigators received approval from their local hospital ethics or institutional review board.

The study aimed at enrolling an unbiased population and sites were encouraged to recruit the first 10 to 20 consecutive eligible patients each month. Data were collected by trained coordinators on standardised case report forms. Demographic characteristics, medical history, presenting symptoms, duration of prehospital delay, biochemical and ECG findings, treatment practices and hospital outcome data were collected. Standardised definitions of all patient-related variables, clinical

\footnotetext{
Abbreviations: CABG, coronary artery bypass grafting; GRACE, Global Registry of Acute Coronary Events; ICTUS, Invasive versus Conservative Treatment in Unstable Coronary Syndromes; NSTEMI, non-ST segment elevation myocardial infarction; $\mathrm{PCl}$, percutaneous coronary intervention; STEMI, ST segment elevation myocardial infarction
} 
diagnoses, and hospital complications and outcomes were used..$^{12}$ All patients were assigned, on the basis of predefined criteria, to one of the following categories: ST segment elevation myocardial infarction (STEMI), non-ST segment elevation myocardial infarction (NSTEMI) or unstable angina. ${ }^{12}$

Patients with STEMI, NSTEMI or unstable angina were characterised on the basis of their discharge diagnosis. STEMI was diagnosed when patients had a new or presumed new ST segment elevation $\geqslant 1 \mathrm{~mm}$ in any location, or new left bundle branch block on the index or qualifying ECG with at least one positive cardiac biochemical marker of necrosis (including troponin measurements, whether qualitative or quantitative). In cases of NSTEMI, at least one positive cardiac biochemical marker of necrosis without new ST segment elevation seen on the index or qualifying ECG had to be present. Unstable angina was diagnosed when serum biochemical markers indicative of myocardial necrosis in each hospital's laboratory were below the diagnostic threshold for infarction. Patients originally admitted because of unstable angina but who developed myocardial infarction during the hospital stay were classified as having a myocardial infarction (please refer to www.outcomes. org/grace for full definitions).

To avoid the possible confounding influence of community hospital referral practice, only hospitals with direct access to a catheterisation laboratory were included in this analysis. Patients were stratified according to their level of risk at presentation according to the GRACE risk model for in-hospital death. ${ }^{14}$

In-hospital outcomes were death; death or myocardial infarction beyond the first $24 \mathrm{~h}$; or reinfarction, stroke (haemorrhagic, non-haemorrhagic or other, defined according to the occurrence of typical neurological signs and symptoms) and major bleeding (defined as life-threatening bleeding requiring transfusion of $\geqslant 2$ units of packed red blood cells or resulting in an absolute decrease in packed cell volume $\geqslant 10 \%$ or death, or haemorrhagic/subdural haematoma). Outcomes that occurred during the six months after hospital discharge were death, unscheduled rehospitalisation for heart disease, myocardial infarction and stroke.

\section{Role of the funding source}

The sponsor, sanofi-aventis, had no involvement in the collection, analysis and interpretation of data; in the writing of the report; and in the decision to submit the paper for publication. The design, conduction and interpretation of GRACE are undertaken by an independent steering committee.

\section{RESULTS}

\section{Patient and hospital characteristics}

A total of 24189 patients with ACS were enrolled in the GRACE registry between April 1999 and September 2004. Table 1 outlines the patients' baseline characteristics according to the GRACE risk score. Data were collected from 73 hospitals with direct access to catheterisation facilities and these were divided into tertiles according to the rates of intervention. A total of 7210 observations were reported for hospitals in the lowest tertile of intervention rates ("low rate"), 7913 for the middle tertile ("medium rate") and 8942 for the highest tertile ("high rate") for percutaneous coronary intervention (PCI) or coronary artery bypass grafting (CABG). Complete data were not available for 124 patients.

The population was stratified into low-, medium- and highrisk tertiles according to the GRACE risk score ${ }^{14}$ (table 1). A gradient of risk characteristics was observed for both non-ST elevation ACS and STEMI.

Table 1 Baseline characteristics according to GRACE risk score for patients presenting with an acute coronary syndrome

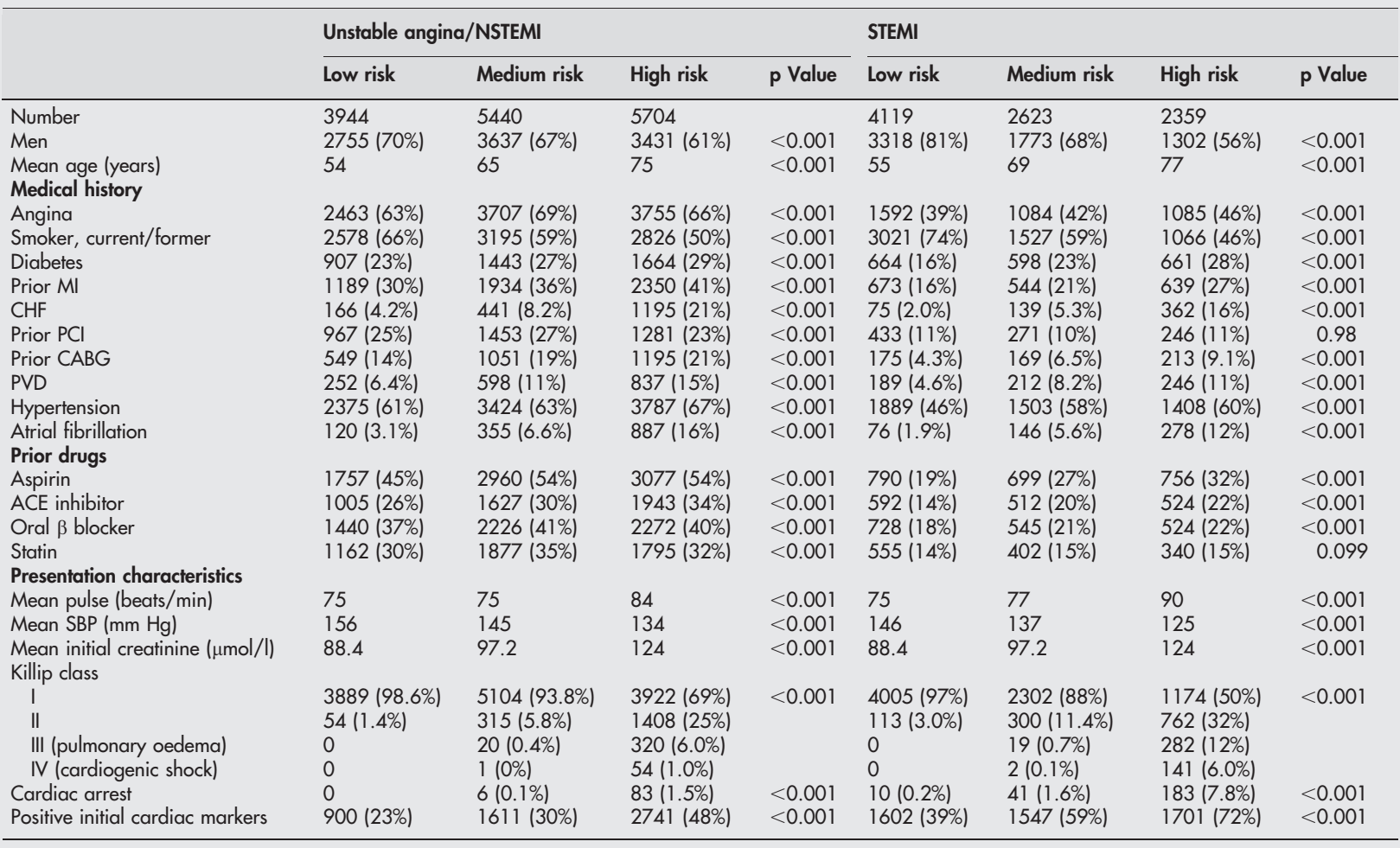

$\mathrm{ACE}$, angiotensin-converting enzyme; $\mathrm{CABG}$, coronary artery bypass grafting; $\mathrm{CHF}$, congestive heart failure; GRACE, Global Registry of Acute Coronary Events; MI myocardial infarction; NSTEMI, non-ST segment elevation myocardial infarction; PCl, percutaneous coronary intervention; PVD, peripheral vascular disease; SBP, systolic blood pressure; STEMI, ST segment elevation myocardial infarction. 


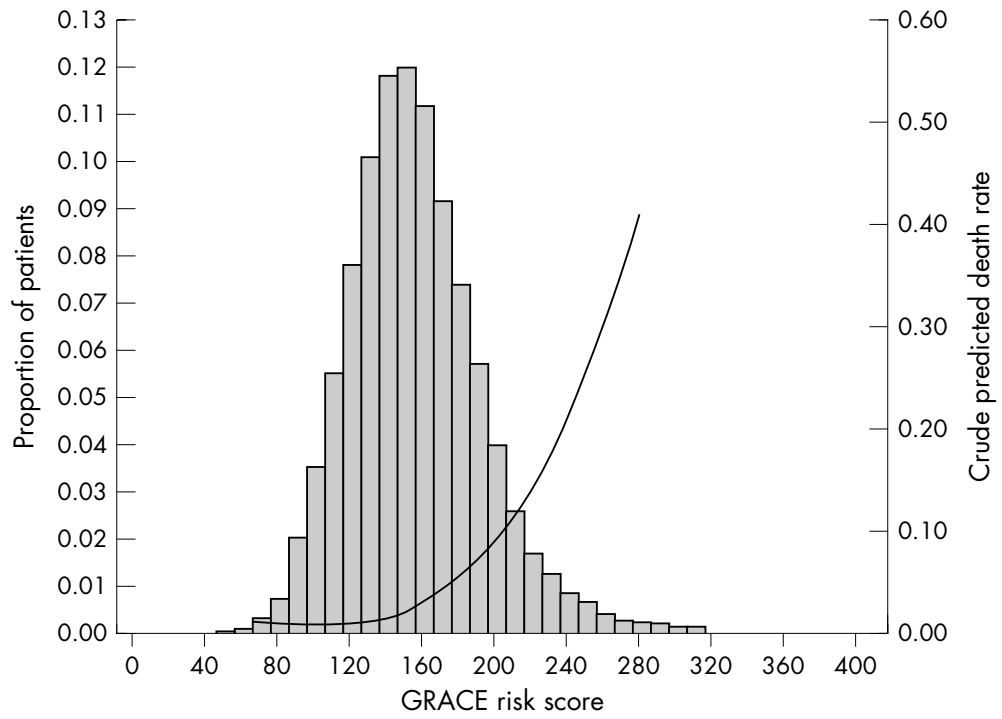

Figure 1 Proportion of patients according to GRACE (Global Registry of Acute Coronary Events) risk score, relation between GRACE risk score and crude predicted death rate (curved line), and use of intervention according to decile of GRACE risk score undergoing intervention (histogram). (Patients with a score $<80$ were aggregated, and those with a rate $>280$ were aggregated; these groups represent $0.6 \%$ and $0.7 \%$ of the data, respectively). Catheterisation, $p=0.0002$; percutaneous coronary intervention (PCI), $p=0.03$; coronary artery bypass grafting $(C A B G), p=0.01$. GRACE risk score adapted from Granger et al ${ }^{14}$.

Determination of non-eligibility for catheterisation, $\mathrm{PCl}$ or $\mathrm{CABG}$ : in-hospital or history of haemorrhagic stroke, and age $>75$ years, renal insufficiency (creatinine $>265 \mu \mathrm{mol} / \mathrm{l}$ ).

Use of intervention according to deciles of GRACE risk score (1 lowest, 10 highest) among potentially eligible patients

GRACE risk score

\begin{tabular}{lcccccccccc} 
& 1 & 2 & 3 & 4 & 5 & 6 & 7 & 8 & 9 & 10 \\
\hline Catheterisation & 53.6 & 52.8 & 53.8 & 52.8 & 50.3 & 50.8 & 53.5 & 50 & 48.4 & 38 \\
PCl & 24.6 & 27 & 26.2 & 24 & 24.4 & 24.2 & 26.2 & 23.5 & 25 & 15.9 \\
CABG & 5.4 & 4.6 & 4.4 & 5.3 & 5.8 & 6.9 & 6.8 & 6.4 & 7.2 & 5.8
\end{tabular}

\section{In-hospital management}

Overall, $32.5 \%$ of patients with NSTEMI or unstable angina underwent PCI and 7.2\% underwent CABG. Rates of revascularisation were high for patients with STEMI $(53.7 \%$ PCI and $4.0 \%$ CABG). The likelihood of a patient undergoing PCI was related to the patient's risk status but the relationship was inverse (fig 1). No such gradient was apparent for patients undergoing CABG. Findings were similar for patients with
STEMI and for non-ST elevation ACS. More high-risk than lowrisk patients underwent echocardiography, but the converse was true for both exercise testing and angiography. More lowrisk (31.2\% primary PCI, $29.5 \%$ fibrinolysis) than medium-risk $(22.7 \%, 18.4 \%$, respectively) and high-risk (15.1\%, 9.2\%, respectively) patients underwent reperfusion (table 2). With the exception of low molecular weight heparin, other antithrombotic drugs were given to more low-risk than high-risk

Table 2 In-hospital management according to GRACE risk score for patients presenting with an acute coronary syndrome

\begin{tabular}{|c|c|c|c|c|c|c|c|c|}
\hline & \multicolumn{4}{|c|}{ Unstable angina/NSTEMI } & \multicolumn{4}{|l|}{ STEMI } \\
\hline & Low risk & Medium risk & High risk & $\mathrm{p}$ Value & Low risk & Medium risk & High risk & p Value \\
\hline Number & 3944 & 5440 & 5704 & & 4119 & 2623 & 2359 & \\
\hline Cardiac catheterisation & $2836(72 \%)$ & $3689(68 \%)$ & 2894 (51\%) & $<0.001$ & $3236(79 \%)$ & 1937 (74\%) & $1342(57 \%)$ & $<0.001$ \\
\hline $\mathrm{PCl}$ & $1554(40 \%)$ & $1907(35 \%)$ & $1426(25 \%)$ & $<0.001$ & $2466(60 \%)$ & $1421(54 \%)$ & $959(41 \%)$ & 0.98 \\
\hline CABG & $298(7.6 \%)$ & 425 (7.9\%) & $361(6.4 \%)$ & 0.006 & $162(4.0 \%)$ & $108(4.2 \%)$ & $86(3.7 \%)$ & 0.67 \\
\hline Fibrinolytics & $113(2.9 \%)$ & $103(1.9 \%)$ & $108(1.9 \%)$ & 0.001 & $1538(38 \%)$ & $828(32 \%)$ & $445(19 \%)$ & $<0.001$ \\
\hline Exercise tolerance test & $977(25 \%)$ & $1205(23 \%)$ & 915 (16\%) & $<0.001$ & $807(20 \%)$ & $414(16 \%)$ & $238(10 \%)$ & $<0.001$ \\
\hline $\begin{array}{l}\text { Echocardiography } \\
\text { In-hospital drugs }\end{array}$ & 2096 (54\%) & $2784(52 \%)$ & $3250(58 \%)$ & $<0.001$ & 2985 (74\%) & $1982(76 \%)$ & 1815 (78\%) & 0.002 \\
\hline Aspirin & 3705 (94\%) & $5080(93 \%)$ & $5136(90 \%)$ & $<0.001$ & 3948 (96\%) & $2490(95 \%)$ & 2147 (91\%) & $<0.001$ \\
\hline Thienopyridine & 2009 (52\%) & $2627(49 \%)$ & $2292(41 \%)$ & $<0.001$ & $2543(62 \%)$ & $1485(57 \%)$ & 1013 (44\%) & $<0.001$ \\
\hline UFH & $1956(50 \%)$ & $2496(47 \%)$ & $2627(47 \%)$ & 0.001 & $2552(63 \%)$ & $1527(59 \%)$ & $1280(55 \%)$ & $<0.001$ \\
\hline LMWH & $2263(58 \%)$ & $3145(58 \%)$ & $3160(56 \%)$ & 0.016 & 2052 (50\%) & $1447(56 \%)$ & $1251(54 \%)$ & $<0.001$ \\
\hline GP Ilb/Illa inhibitor & $1008(26 \%)$ & $1197(22 \%)$ & $1002(18 \%)$ & $<0.001$ & $1669(41 \%)$ & $914(35 \%)$ & $659(28 \%)$ & 0.099 \\
\hline ACE inhibitor & $2157(55 \%)$ & 2911 (54\%) & 3234 (57\%) & 0.004 & $2796(68 \%)$ & $1861(71 \%)$ & $1589(68 \%)$ & 0.01 \\
\hline$\beta$ blocker & $3352(85 \%)$ & 4533 (84\%) & 4251 (75\%) & $<0.001$ & $3706(90 \%)$ & 2217 (85\%) & $1615(69 \%)$ & $<0.001$ \\
\hline Calcium antagonist & $1120(29 \%)$ & $1745(32 \%)$ & 1936 (34\%) & $<0.001$ & $578(14 \%)$ & 490 (19\%) & 425 (18\%) & $<0.001$ \\
\hline
\end{tabular}

ACE, angiotensin-converting enzyme; CABG, coronary artery bypass grafting; GP, glycoprotein; GRACE, Global Registry of Acute Coronary Events; LMWH, low molecular weight heparin; MI, myocardial infarction; NSTEMI, non-ST segment elevation myocardial infarction; PCl, percutaneous coronary intervention; STEMI, ST segment elevation myocardial infarction; UFH, unfractionated heparin. 

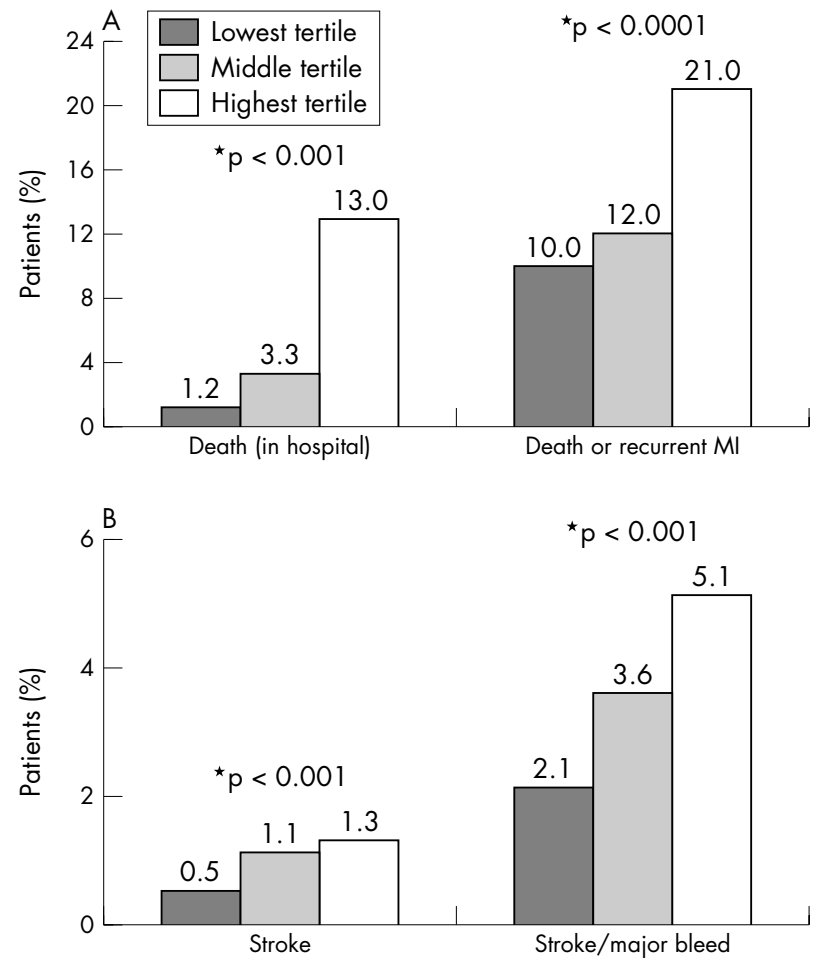

Figure 2 (A) Frequency of death and recurrent myocardial infarction (MI) by tertile of GRACE (Global Registry of Acute Coronary Events) risk score in patients with ST or non-ST segment elevation myocardial infarction. (B) Frequency of stroke and major bleeding by tertile of GRACE risk score in patients with ST or non-ST segment elevation myocardial infarction. ${ }^{*} p$ values across three tertiles.

patients (table 2). Thienopyridines, unfractionated heparin and glycoprotein IIb/IIIa inhibitors were all given to more low- than medium-risk patients, consistent with the gradient in rates of angiography and PCI (table 2).

\section{In-hospital outcomes}

The rates of in-hospital death among low-, medium- and highrisk categories of patients were highly consistent with those predicted by the GRACE risk model ${ }^{14}$ (3.0\% observed death rate for patients with non-ST elevation ACS $v 3.8 \%$ predicted; $7.0 \%$ observed death rate for patients with STEMI $v 5.3 \%$ predicted). The risk of death ranged from $1.2 \%$ for low-risk to $3.3 \%$ for medium-risk and $13.0 \%$ for high-risk patients. Fig 1 shows the proportions of patients enrolled in GRACE according to their risk score and the relation between risk score and crude predicted death rate. The incidence of in-hospital stroke was greatest among high-risk patients with STEMI treated at hospitals with a high rate of intervention $(1.8 \%)$ compared with hospitals with medium $(1.4 \%)$ or low $(1.6 \%)$ rates of intervention. The incidence of stroke was lower among patients with NSTEMI than among those with STEMI $(0.7 \% v 1.2 \%$ in high-risk patients, and $0.3 \% \vee 1.0 \%$ in low- to medium-risk patients).

Fig 2A shows the relationship between patients' baseline risk status (in tertiles) and the observed rates of in-hospital death, and death or myocardial infarction beyond the first $24 \mathrm{~h}$ or reinfarction. Similarly, fig 2B shows a direct relationship between baseline risk and increasing frequency of stroke and major bleeding.

To determine whether the relationship between risk and rates of revascularisation was influenced by whether hospitals
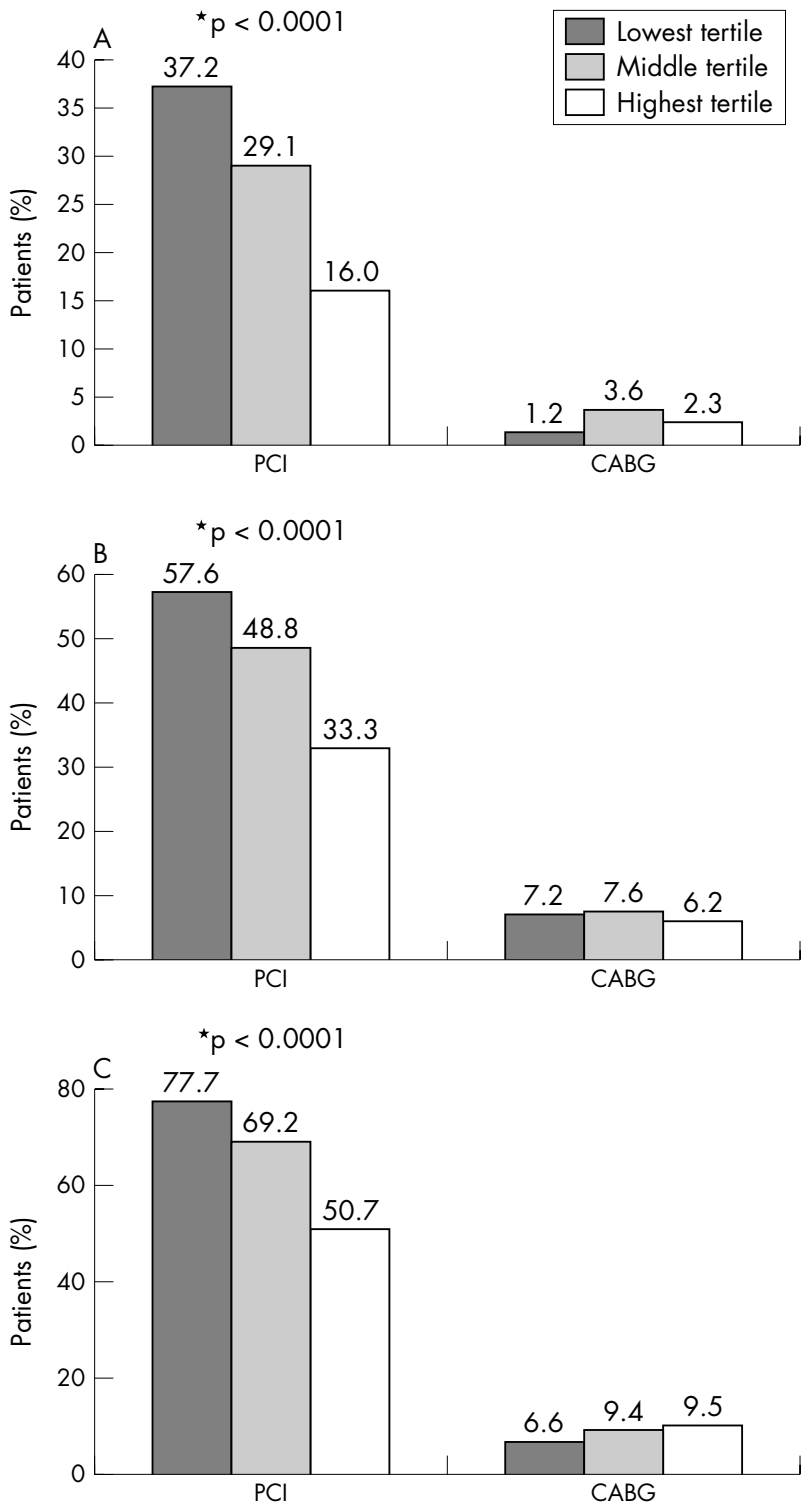

Figure 3 Frequency of intervention by tertile of GRACE (Global Registry of Acute Coronary Events) risk score according to hospitals with (A) low (53\%), (B) medium (73\%) and (C) high (88\%) rates of angiography in patients with ST or non-ST segment elevation myocardial infarction. * $p$ values across three tertiles.

adopted a more or less aggressive angiographic strategy, hospitals were divided into tertiles according to rates of angiography (53\% low, $73 \%$ medium and $88 \%$ high rates). The inverse relationship between risk status of the patient at presentation and likelihood of proceeding to PCI was independent of whether hospitals adopted more or less aggressive angiography strategies (fig 3). Further, the findings were consistent across geographic regions and healthcare systems.

\section{DISCUSSION}

On the basis of evidence from randomised trials of interventional versus conservative strategies in $\mathrm{ACS},{ }^{1-5}$ European and North American guidelines advocate revascularisation for moderate- or higher-risk patients, but not for low-risk patients. ${ }^{9}{ }^{10}$ Similarly, in studies of STEMI, subgroup analysis shows that the absolute benefits of revascularisation are highest among patients with more extensive infarction. ${ }^{15}$ 
Clinical practice would therefore be expected to reflect this evidence. In contrast, we saw the opposite in our study. We observed an inverse relationship between the rate of PCI (or the rate of angiography) and the risk status of the patient, irrespective of whether the patient had unstable angina, NSTEMI or STEMI. We saw no such inverse relationship between risk status and rate of $\mathrm{CABG}$, suggesting that different criteria are applied in the decision to proceed to CABG or to PCI. Higher-risk patients were as likely as lower-risk patients to be referred for $\mathrm{CABG}$. In most instances the interventionist undertaking the diagnostic angiography would have made the decision to proceed to $\mathrm{PCI},{ }^{16}$ whereas the decision to perform CABG would have required discussion with cardiac surgeons. The striking inverse relationship between risk status and performance of angiography ( $\mathrm{p}<0.001$; table 2 ) indicates that clinicians responsible for the referral process apply a risk-averse strategy before obtaining information from coronary angiography. With the exception of low molecular weight heparin, antithrombotics were given to more low-risk than medium- or high-risk patients, consistent with the rates of angiography and PCI.

Patients with more extensive co-morbidity may be expected not to be candidates for CABG, and a proportion of such patients would not be candidates for PCI. However, the justification for the higher rate of PCI among low-risk patients than among medium-risk patients is not apparent. The issue has been discussed in the context of the operator's decision to proceed to PCI on the basis of findings at angiography. ${ }^{16}$ In fig 1 , the results are analysed according to deciles of GRACE risk score ${ }^{14}$ limited to patients without renal insufficiency (creatinine $>265 \mu \mathrm{mol} / \mathrm{l}$ ) and excluding patients older than 75 years (as a surrogate for co-morbidity). The relation between risk status and angiography, PCI or CABG was not altered by these exclusions.

In settings where rates of angiography are lower, perhaps related to availability of personnel and resources, a more selective strategy for PCI might have been expected to be adopted (favouring those at higher risk of death or myocardial infarction). However, the relationship is no different from that in hospitals with medium or high rates of angiography. The findings suggest that a risk-averse strategy is widely adopted for angiography in ACS. The findings are consistent, irrespective of whether sites adopt a more or less aggressive strategy for revascularisation and irrespective of healthcare system and geographic region. As fig 3 shows, hospitals in the highest tertile for rates of angiography have PCI rates more than twofold greater than those in the lowest tertile for respective risk groups (high risk $77.7 \%$ v 37.2\% PCI; medium risk $69.2 \% v$ $29.1 \%$; low risk $50.7 \% \vee 16.0 \%$ ). Hence, if patient contraindications were responsible for the findings, this would not explain how the more aggressive centres undertake much higher rates of angiography and intervention than less aggressive centres for risk-matched patients. The consistency of inverse relationship between risk and invasive procedures suggests that the observations are widely applicable, rather than determined by resource allocation or staff availability. Paradoxically, this pattern of practice is not supported by trial evidence $^{1-7}$ or by guidelines for clinical practice. ${ }^{10}$

Although we excluded patients first admitted to community hospitals from our analysis, our findings may provide some explanation for those of Van de Werf et al. ${ }^{17}$ In their study of patients enrolled in the GRACE database, patients admitted to hospitals with catheterisation facilities were more likely to undergo intervention than were patients admitted to sites without such facilities, but they had a higher risk of death within six months of discharge. This later risk may reflect hazards of intervention (but very modest gain) among low-risk patients. The randomised trial evidence and the guidelines support the use of revascularisation in moderate- or high-risk patients, irrespective of the presence of on-site catheterisation facilities.

\section{Strengths and limitations}

GRACE is the largest multinational registry to study the complete spectrum of patients with an ACS. Standardised criteria are used for defining ACS and hospital outcomes, and rigorous quality control and audit measures are used. Nevertheless, the GRACE registry shares the limitations of observational studies. This analysis is confined to centres with direct access to angiographic facilities and therefore excludes patients who presented to district or community hospitals without interventional facilities. Thus, the findings are not confounded by issues relating to interhospital transfer of patients. Restriction of the registry to patients who are admitted may have resulted in the exclusion of patients dying early in the emergency room, but such patients are very unlikely to be selected for revascularisation. The participating clusters reflect regional practices and outcomes but do not necessarily reflect practice for specific countries. Among the highest-risk patients a significant proportion may not be technically suitable for revascularisation (for example, in the highest decile of risk; fig 2) and in some patients the balance of co-morbidity may be such that revascularisation may not offer an acceptable level of periprocedural risk. However, this cannot explain the lower rates of PCI among medium than among lowrisk patients or the highly significant $(p=0.0008)$ inverse relationship between rates of angiography and risk status (table 2).

In conclusion, systematic and accurate risk stratification may allow higher-risk patients to be selected for revascularisation procedures, in contrast to current international practice.

\section{ACKNOWLEDGEMENTS}

The authors thank the physicians and nurses who are participating in GRACE. We thank Dr Sophie Rushton-Smith, who provided editorial assistance and was funded by sanofi-aventis.

To find out more about GRACE, and to download the GRACE risk score, visit the website at www.outcomes.org/grace.

GRACE Steering Committee and Co-Chairmanship: Keith A A Fox, Joel M Gore (Co-Chairs); Kim A Eagle, P Gabriel Steg (Publication Committee Co-Chairs); Giancarlo Agnelli, Frederick A Anderson Jr, Álvaro Avezum, David Brieger, Andrzej Budaj, Marcus D Flather, Robert J Goldberg, Shaun G Goodman, Christopher B Granger, Dietrich C Gulba, Enrique P Gurfinkel, Brian M Kennelly, Werner Klein (deceased), José López-Sendón, Gilles Montalescot and Frans Van de Werf.

\section{Authors' affiliations}

K A A Fox, Cardiovascular Research, Division of Medical \& Radiological Sciences, The University of Edinburgh, Edinburgh, UK

F A Anderson, O H Dabbous, Center for Outcomes Research, University of Massachusetts Medical School, Worcester, Massachusetts, USA

P G Steg, Cardiology, Hôpital Bichat, Paris, France

J López-Sendón, Department of Cardiology, Hospital Universitario La Paz, Madrid, Spain

F Van de Werf, Department of Cardiology, Universitair Ziekenhuis

Gasthuisberg, Leuven, Belgium

A Budaj, Postgraduate Medical School, Grochowski Hospital, Warsaw, Poland

E P Gurfinkel, Department of Cardiology, ICYCC Favaloro Foundation, Buenos Aires, Argentina

S G Goodman, Canadian Heart Research Centre and Terrence Donnelly Heart Centre, Division of Cardiology, St Michael's Hospital, University of Toronto, Toronto, Ontario, Canada

D Brieger, Coronary Care Unit, Concord Hospital, Sydney, Australia

GRACE is supported by an unrestricted educational grant from sanofiaventis, Paris, France. 
Competing interests: KAAF: British Heart Foundation, Medical Research Council, Wellcome Trust, sanofi-aventis; FAA: sanofi-aventis; OHD: no conflicts to disclose; PGS: sanofi-aventis; JL-S: sanofi-aventis, Pfizer, the TIMI group, BMS, Medtronic and Guidant; FV: Boehringer Ingelheim, sanofi-aventis, Proctor and Gamble, Servier, Novartis, MSD and Schering Plough; A Budaj: sanofi-aventis; EPG: sanofi-aventis, Lilly and Astra Zeneca; SGG: sanofi-aventis, AstraZeneca, Boehringer Ingelheim, BristolMyers Squibb, Glaxo Smith Kline, Hoffmann-LaRoche Pharmaceuticals, Merck \& Co, Inc, Novartis, Pfizer Inc, Sanofi-Synthelabo, Schering Corporation, Millennium Pharmaceuticals, Inc and The Medicines Company; DB: sanofi-aventis.

Author contributors: Conception and design: KAA Fox, FA Anderson, PG Steg, J López-Sendón, F Van de Werf, A Budaj, EP Gurfinkel, SG Goodman, D Brieger; analysis and interpretation of the data: KAA Fox, OH Dabbous, PG Steg, J López-Sendón, F Van de Werf, A Budaj, EP Gurfinkel, SG Goodman, D Brieger; drafting of the article: KAA Fox, OH Dabbous; critical revision of the article for important intellectual content: KAA Fox, FA Anderson, OH Dabbous, PG Steg, J López-Sendón, F Van de Werf, A Budaj, EP Gurfinkel, SG Goodman, D Brieger; final approval of the article: KAA Fox, FA Anderson, OH Dabbous, PG Steg, J LópezSendón, F Van de Werf, A Budaj, EP Gurfinkel, SG Goodman, D Brieger; statistical expertise: $\mathrm{OH}$ Dabbous; obtaining funding: FA Anderson; collection and assembly of data: KAA Fox, PG Steg, J López-Sendón, F Van de Werf, A Budaj, EP Gurfinkel, SG Goodman, D Brieger.

\section{REFERENCES}

1 Keeley EC, Boura JA, Grines CL. Primary angioplasty versus intravenous thrombolytic therapy for acute myocardial infarction: a quantitative review of 23 randomised trials. Lancet 2003;361:13-20.

2 FRISC II Investigators. Invasive compared with non-invasive treatment in unstable coronary-artery disease: FRISC II prospective randomised multicentre study. Lancet 1999:354:708-15.

3 Cannon CP, Weintraub WS, Demopoulos LA, et al. Comparison of early invasive and conservative strategies in patients with unstable coronary syndromes treated with the glycoprotein Ilb/llla inhibitor tirofiban. N Engl J Med 2001;344:1879-87.

4 Fox KA, Poole-Wilson PA, Henderson RA, et al. Interventional versus conservative treatment for patients with unstable angina or non-ST-elevation myocardial infarction: the British Heart Foundation RITA 3 randomised trial. Randomized Intervention Trial of unstable Angina. Lancet 2002;360:743-51.
5 Mehta SR, Cannon CP, Fox KA, et al. Routine vs selective invasive strategies in patients with acute coronary syndromes: a collaborative meta-analysis of randomized trials. JAMA 2005;293:2908-17.

6 Bhatt DL, Roe MT, Peterson ED, et al. Utilization of early invasive management strategies for high-risk patients with non-ST-segment elevation acute coronary syndromes: results from the CRUSADE Quality Improvement Initiative. JAMA 2004;292:2096-104

7 Fox KA, Poole-Wilson P, Clayton TC, et al. 5-year outcome of an interventional strategy in non-ST-elevation acute coronary syndrome: the British Heart Foundation RITA 3 randomised trial. Lancet 2005;366:914-20.

8 De Winter RJ, Windhausen F, Cornel JH, et al. Early invasive versus selectively invasive management for acute coronary syndromes. N Engl J Med 2005;353:1095-104

9 Bertrand ME, Simoons ML, Fox KA, et al. Management of acute coronary syndromes in patients presenting without persistent ST-segment elevation. The Task Force on the management of acute coronary syndromes of the European Society of Cardiology. Eur Heart J 2002;23:1809-40.

10 Braunwald E, Antman EM, Beasley JW, et al. ACC/AHA 2002 guideline update for the management of patients with unstable angina and non-ST-segment elevation myocardial infarction-summary article: a report of the American College of Cardiology/American Heart Association task force on practice guidelines (Committee on the Management of Patients With Unstable Angina) J Am Coll Cardiol 2002:40:1366-74.

11 Eagle KA, Goodman SG, Avezum A, et al. Practice variation and missed opportunities for reperfusion in ST-segment-elevation myocardial infarction: findings from the Global Registry of Acute Coronary Events (GRACE). Lancet 2002;359:373-7

12 The GRACE Investigators. Rationale and design of the GRACE (Global Registry of Acute Coronary Events) Project: a multinational registry of patients hospitalized with acute coronary syndromes. Am Heart J 2001;141:190-9.

13 Steg PG, Goldberg RJ, Gore JM, et al. Baseline characteristics, management practices, and in-hospital outcomes of patients hospitalized with acute coronary syndromes in the Global Registry of Acute Coronary Events (GRACE). Am J Cardiol 2002;90:358-63.

14 Granger CB, Goldberg RJ, Dabbous O, et al. Predictors of hospital mortality in the Global Registry of Acute Coronary Events. Arch Intern Med 2003;163:2345-53.

15 FTT Collaborative Group. Indications for fibrinolytic therapy in suspected acute myocardial infarction: collaborative overview of early mortality and major morbidity results from all randomised trials of more than 1000 patients. Fibrinolytic Therapy Trialists' (FTT) Collaborative Group. Lancet 1994;343:311-22

16 Topol EJ, Nissen SE. Our preoccupation with coronary luminology: the dissociation between clinical and angiographic findings in ischemic heart disease. Circulation 1995;92:2333-42.

17 Van de Werf F, Gore JM, Avezum A, et al. Access to catheterisation facilities in patients admitted with acute coronary syndrome: multinational registry study. BMJ 2005;330:441. 